\title{
Tandem electrospinning for heterogeneous nanofiber patterns
}

Citation for published version (APA):

Wieringa, P., Truckenmuller, R., Micera, S., van Wezel, R., \& Moroni, L. (2020). Tandem electrospinning for heterogeneous nanofiber patterns. Biofabrication, 12(2), [025010]. https://doi.org/10.1088/1758$5090 / a b 6 e d b$

Document status and date:

Published: 01/04/2020

DOI:

10.1088/1758-5090/ab6edb

Document Version:

Publisher's PDF, also known as Version of record

Document license:

Taverne

Please check the document version of this publication:

- A submitted manuscript is the version of the article upon submission and before peer-review. There can be important differences between the submitted version and the official published version of record.

People interested in the research are advised to contact the author for the final version of the publication, or visit the DOI to the publisher's website.

- The final author version and the galley proof are versions of the publication after peer review.

- The final published version features the final layout of the paper including the volume, issue and page numbers.

Link to publication

\footnotetext{
General rights rights.

- You may freely distribute the URL identifying the publication in the public portal. please follow below link for the End User Agreement:

www.umlib.nl/taverne-license

Take down policy

If you believe that this document breaches copyright please contact us at:

repository@maastrichtuniversity.nl

providing details and we will investigate your claim.
}

Copyright and moral rights for the publications made accessible in the public portal are retained by the authors and/or other copyright owners and it is a condition of accessing publications that users recognise and abide by the legal requirements associated with these

- Users may download and print one copy of any publication from the public portal for the purpose of private study or research.

- You may not further distribute the material or use it for any profit-making activity or commercial gain

If the publication is distributed under the terms of Article $25 \mathrm{fa}$ of the Dutch Copyright Act, indicated by the "Taverne" license above, 


\section{PAPER}

Tandem electrospinning for heterogeneous nanofiber patterns

To cite this article: Paul Wieringa et al 2020 Biofabrication 12025010

\section{Recent citations}

A One-Step Biofunctionalization Strategy of Electrospun Scaffolds Enables Spatially Selective Presentation of Biological Cues Paul Wieringa et al

View the article online for updates and enhancements.

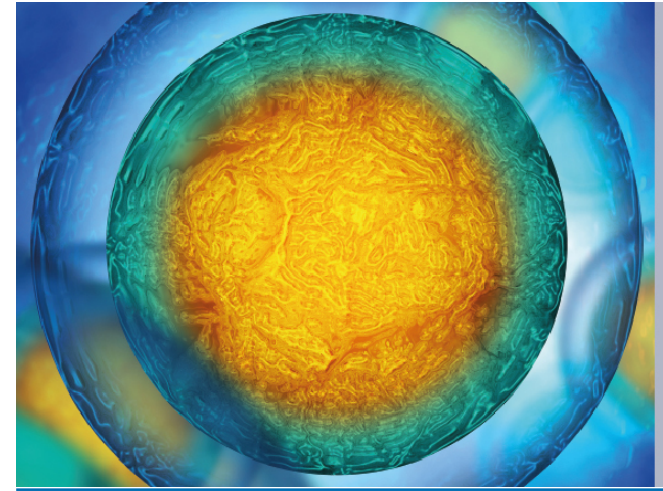

Biophysical Society $\quad$ IOP $\mid$ ebooks $^{\text {'m }}$

$\overline{\equiv \equiv}$

Your publishing choice in all areas of biophysics

research.

Start exploring the collection-download the first chapter of every title for free. 


\title{
Biofabrication
}

\section{PAPER}

\section{Tandem electrospinning for heterogeneous nanofiber patterns}

RECEIVED

13 September 2019

REVISED

15 January 2020

ACCEPTED FOR PUBLICATION

22 January 2020

PUBLISHED

13 February 2020

\author{
Paul Wieringa ${ }^{1,2,3,4,7} \oplus$, Roman Truckenmuller ${ }^{1,7}$, Silvestro Micera ${ }^{3,5}$, Richard van Wezel ${ }^{2,6}$ and \\ Lorenzo Moroni ${ }^{1,7,8}$ (1) \\ 1 Department of Tissue Regeneration, MIRA Institute for Biomedical Technology and Technical Medicine, University of Twente, The \\ Netherlands \\ 2 Department of Biomedical Signals and Systems, MIRA Institute for Biomedical Technology and Technical Medicine, Twente University, \\ Enschede, The Netherlands \\ 3 BioRobotics Institute-Scuola Superiore Sant'Anna, Pisa, Italy \\ 4 Istituto Italiano di Tecnologia, Center for Micro-BioRobotics @SSSA, Viale Rinaldo Piaggio 34, I-56025 Pontedera, Pisa, Italy \\ 5 Translational Neural Engineering Laboratory, Center for Neuroprosthetics and Institute of Bioengineering, School of Engineering, Ecole \\ Polytechnique Federale de Lausanne, Lausanne, Switzerland \\ 6 Biophysics, Donders Institute for Brain, Cognition and Behaviour, Radboud University, Nijmegen, The Netherlands \\ 7 Department of Complex Tissue Regeneration, MERLN Institute for Technology Inspired Regenerative Medicine, Maastricht University, \\ The Netherlands \\ 8 Author to whom any correspondence should be addressed. \\ E-mail:1.moroni@maastrichtuniversity.nl
}

Keywords: electrospinning, patterns, cell adhesion

Supplementary material for this article is available online

\begin{abstract}
Smart nanofibrillar constructs can be a promising technological solution for many emerging and established fields, facilitating the potential impact of nano-scale strategies to address relevant technological challenges. As a fabrication technique, electrospinning (ESP) is relatively well-known, accessible, economic, and fast, though until now has shown limitation over control and design of the fibrillar constructs which can be produced. Here, we introduce 'Tandem Electrospinning' (T-ESP), a novel technique able to create increasingly complex patterns of fibrous polymeric constructs on a micro and nano-scale. Modifying a standard ESP configuration results in a focusing electric field that is able to spatially define the deposition pattern of multiple polymer jets simultaneously. Additional spatially defined heterogeneity is achieved by tuning polymer solution properties to obtain a gradient of fiber alignment. Heterogeneous fibrous meshes are created with either random, aligned, or a divergent fiber patterns. This approach holds potential for many fields, with application examples shown for tissue engineering and separation technologies. The technique outlined here provides a rapid, versatile, and accessible method for polymeric nanofabrication of patterned heterogeneous fibrous constructs. Polymer properties are also shown to dictate fiber alignment, providing further insight into the mechanisms involved in the electrospinning fabrication process.
\end{abstract}

\section{Introduction}

Recent years have seen a renewed interest in electrospinning (ESP) for micro- and nano-scale fabrication, driven by new applications in the fields of environment and security, such as filtration or particle sensing, biomedical technologies, in form of tissue scaffolds or drug release platforms, and in the energy sector, with potential for improving both solar and fuel cell technologies [1-6]. The promise of creating micro- and nano-sized fibers in a simple, relatively inexpensive and high-throughput manner has spurred development of ESP fabrication techniques, leading to the production of fibrous meshes with high surface area and tunable porosity.

Conventional ESP results in a chaotic fibrous mesh, narrowing the application of this form of ESP to areas such as textile and filtration industries. Strategies have been developed to produce fiber constructs useful for other applications, but these approaches still exhibit limitations in terms of spatially defined fiber deposition and orientation, and typically produce simple polymer constructs consisting of a single fiber type. ESP across a planar target gap electrode or 
patterned target electrodes produces simple parallel fibers or cross-hatched fiber patterns, respectively, with limited spatial definition and restricted to single fiber composition or layer-by-layer fiber assemblies [1, 7-11]. In general, deposition and spatial control of multiple polymer fibers is hampered by the mutual repulsion of multiple charged jets [12-14]. Attempts to create multimodal fiber constructs have relied on the chaotic fluctuations of the 'whipping' polymer jets to achieve overlap between fiber populations, intrinsically precluding any form of fiber orientation. ESP onto a rotating mandrel also produces simple parallel fibers, with increased complexity possible by simultaneously electrospinning different polymers onto a rotating mandrel [15]. However, more elaborate, spatially defined multimodal patterns are not possible. The most detailed fiber patterns have been created by near-field electrospining (NFES) or melt electrospinning, which are less accessible and unable to simultaneously deposit multiple fiber types within a confined region $[16,17]$.

Here, we describe the development of T-ESP techniques, which allow for simultaneous electrospinning of up to three polymer fibers. Using modifications to a traditional ESP setup, we can achieve a high degree of control of planar ESP patterning. Control over multiple jets is achieved by manipulating the electrostatic field, resulting in the creation of ESP multi-material fibrous constructs. This allows ESP fiber patterns of intermingled, heterogeneous fiber populations in random, parallel or divergent ('Y-shaped') orientations. Finally, we show how this facile and economic fabrication method can be used to mimic branched structures found in biological systems and present an example of a divergent tissue scaffold supporting spatially defined differences in neurite outgrowth.

\section{Experimental section}

\section{Polymer solution preparation and characterization} All polymers were dissolved in associated solvent solutions overnight. The PEOT/PBT polymers were prepared in a solvent blend of chloroform and hexafluoroisopropanol (HFIP; see supplementary material, tables S1 and S2 are available online at stacks. iop.org/BF/12/025010/mmedia for a details on polymers and solutions) and appropriate dyes were added at $0.01 \% \mathrm{w} / \mathrm{v}$. Specifically, a $20 \% \mathrm{w} / \mathrm{v}$ solution of 300PEOT55PBT45 polymer was prepared and designated HighE for its small molecular entanglement weight $\left(M_{e}\right)$ of $250 \mathrm{Da}$, a $20 \% \mathrm{w} / \mathrm{v}$ solution of 300PEOT70PBT30 was prepared and labelled MidE $\left(M_{e}: 450 \mathrm{Da}\right)$, and polymer solutions of 1000PEOT70PBT30 $\left(M_{e}: 710 \mathrm{Da}\right)$ were prepared at either $20 \% \mathrm{w} / \mathrm{v}$ (LowE) or $15 \% \mathrm{w} / \mathrm{v}\left(\operatorname{LowE}_{m v} ; m v\right.$ : matched viscosity). Where applicable, $\mathrm{NaCl}$ (Sigma Aldrich $\mathrm{GmbH}$, Germany) was added to polymer solutions at final concentration of $0.05 \mathrm{mg} \mathrm{ml}^{-1}$ and polymer solutions were labelled with a ' + ' (e.g. LowE $\mathrm{E}^{+}$). Conductivity was measured using a custom gold parallel plate apparatus at $20{ }^{\circ} \mathrm{C}$ (see supplementary material S12 for full description) and viscosity was measured using a Brookfield DV-E with an s21 mandrel at $30^{\circ} \mathrm{C}$ and $100 \mathrm{rpm}$.

\section{Electrospinning for T-ESP development}

A custom ESP setup was used with an environmental chamber $\left(25^{\circ} \mathrm{C}, 30 \%\right.$ humidity $)$ and a large upper parallel plate $(30 \mathrm{~cm}$ by $20 \mathrm{~cm}$ ). ESP was performed at $25 \mathrm{KV}$, a working distance of $20 \mathrm{~cm}$, a flow rate of $1 \mathrm{ml} \mathrm{h}^{-1}$ and for an ESP interval of $1 \mathrm{~min}$. For tandem ESP and triple ESP, needles were mounted along the $Y$ axis of the collector target (figure 1(B)) at an interneedle separation of $1 \mathrm{~cm}$ and $5 \mathrm{~cm}$, respectively. A $14 \mathrm{~mm}$ coverslip (Menzel-Glaser) was the target substrate used to collect deposited fibers, placed on the collector electrodes of either a parallel or epsilon arrangement (see supplementary figure S8).

\section{Electrospinning T-ESP scaffolds for specific applications}

Cell scaffolds were prepared using the epsilon electrode configuration shown in Figure S9. In lieu of coverslips for improved cell culture handling, fibers were deposited onto a mesh support ring with an outer diameter $15 \mathrm{~mm}$ and an inner aperture diameter of $12 \mathrm{~mm}$, made from a nonwoven sheet of polyester (6691 LL (40 $\mathrm{g} \mathrm{m}^{-2}$ ), a kind gift from Lantor B.V., the Netherlands). A working distance of $20 \mathrm{~cm}$ and a voltage of $20 \mathrm{KV}$ was used for an ESP time of $30 \mathrm{~s}$. To ensure jet stability, the HighE solution had a flow rate of $1 \mathrm{ml} \mathrm{h}^{-1}$ and a flow rate of $0.5 \mathrm{ml} \mathrm{h}^{-1}$ for the HighE-collagen solution. Parallel T-ESP scaffolds for liquid phase separation were prepared with the standard gap electrode configuration using an HighE solution $\left(1.0 \mathrm{ml} \mathrm{h}^{-1}\right)$ and a $10 \%$ PVA solution $\left(0.5 \mathrm{ml} \mathrm{h}^{-1}\right)$ on glass coverslips. Scaffold were immersed in methanol with 5\% paraformaldehyde for $24 \mathrm{~h}$ to crosslink the PVA fibers and then air dried for $24 \mathrm{~h}$ before use.

\section{Analysis of ESP process}

Images of whipping were captured by a Luminix DMC G3 and fiber pattern images were stitched together using a Nikon Eclipse Ti with an automated stage at $10 \times$ magnification. Fibers were gold sputter-coated and examined with a XL 30 ESEM-FEG (Phillips) operating at $10 \mathrm{kV}$. A minimum of 100 fibers were measured per population and minimum of 5 images per fiber population were evaluated for fiber orientation using the OrientationJ plugin [18], providing a coherence value between 0 (isotropic) and 1 (perfectly anisotropic). Fiber orientation was also evaluated by creating an FFT image of SEM images [19]. 

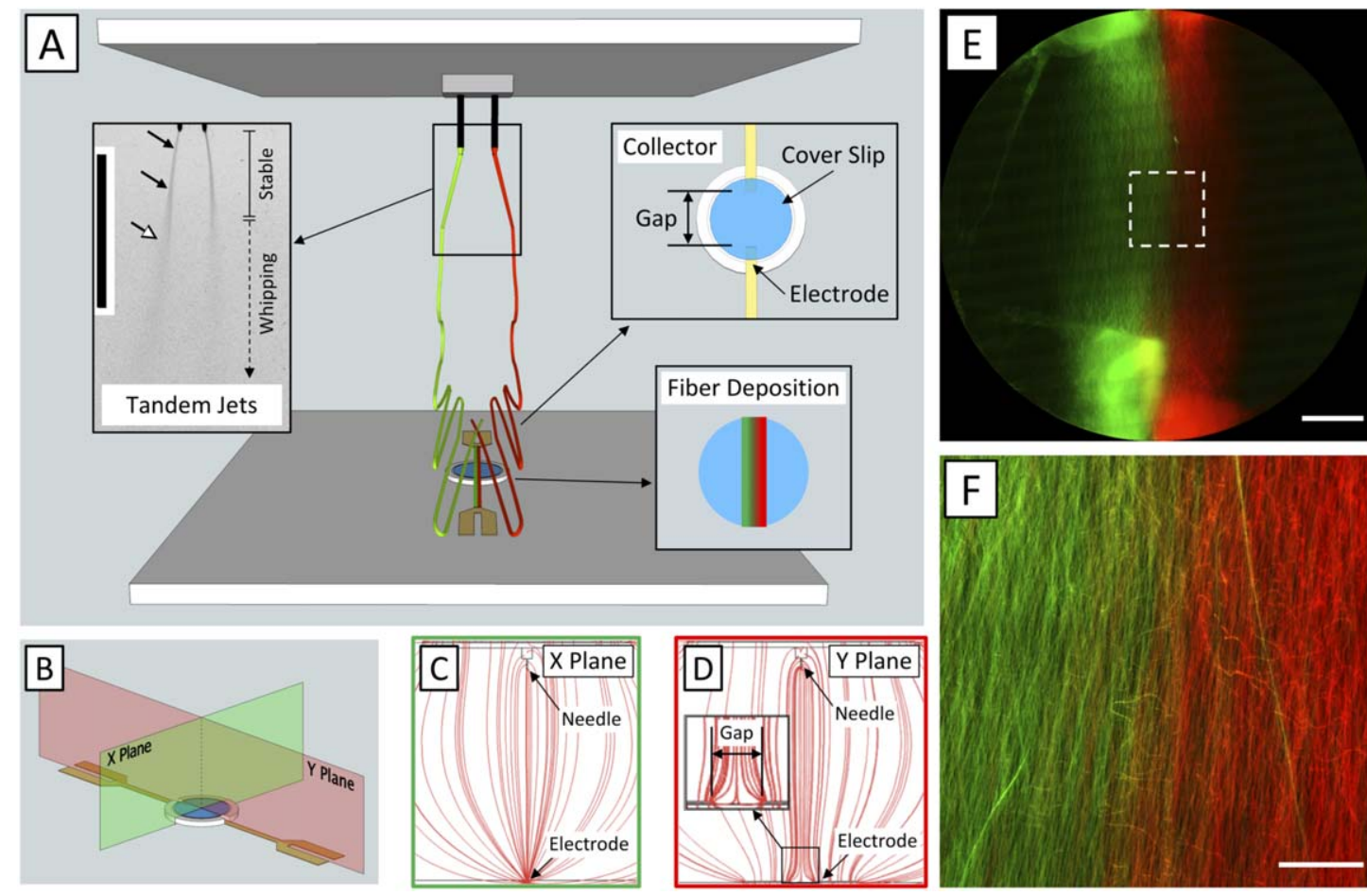

Figure 1. The Tandem ESP Apparatus. (A) A depiction showing an upper parallel plate to which 2 needles are mounted and high voltage is applied and a base plate with a gap electrode arrangement (detail shown in Inset: 'Collector'). A 10 mm gap was made between two $2 \mathrm{~mm}$ wide electrodes. Shown is the placement of a $14 \mathrm{~mm}$ glass coverslip on top of the two electrodes, used to collect fibers patterned across the gap (Inset: 'Fiber Deposition'). ESP with single needles were centered over the coverslip. Tandem and triple ESP needles were arranged along the $X$ axis (see $B$ for orientation). Also shown is a representation of two simultaneous jets (red and green) as well as an image of the polymer jets of two HighE polymer solutions (Inset: 'Tandem Jets'; scale bar: $5 \mathrm{~cm}$ ); dark arrows indicate the stable jet phase and a white arrow indicates the onset of the whipping phase, where the jet becomes too small to be easily visible. (B) The gap electrode configuration was modeled to show the electric field generation in both the $X$ and $Y$ plane. (C) The electric field in the $X$ plane of the gap electrode configuration shows the focusing electric field this electrode arrangement creates. (D) The same electric field in the orthogonal $Y$ plane clearly shows the divergent electric field (Inset: 'Gap') which causes fibers to align across the gap. (E) The tandem ESP deposition pattern of two identical HighE polymer solutions with Macrolex ${ }^{\oplus e d}$ G and Green dyes added, respectively (scale bar: $2 \mathrm{~mm}$ ). This particular polymer solution produces consistent electrospun fibers (green:

$0.653 \pm 0.143 \mu \mathrm{m}$; red: $0.637 \pm 0.136 \mu \mathrm{m}$ ) and achieves a high degree of alignment when using a gap target electrode configuration (green: $0.734 \pm 0.115$; red:0.817 \pm 0.097 ). (F) A magnified view of the overlapping regions between the two fiber populations is shown, clearly depicting the defined yellow gradient transition from red to green (scale bar: $500 \mu \mathrm{m}$ ).

\section{Cell culture}

Dorsal root ganglia (DRGs) were explanted from $2 \mathrm{~d}$ old rat pups (Wistar Unilever: HsdCpb:WA). All procedures followed national and European laws and guidelines and were approved by the local ethical committee. Briefly, rats were sacrificed by cervical dislocation under general anaesthesia (4\% Isoflurane) and then decapitated. Individual ganglia were removed from the spinal column and nerve roots were stripped under aseptic conditions with the aid of a stereomicroscope. DRGs were cut in half to expose interior neurites and placed on the fibrous ESP scaffolds. For divergent T-ESP scaffolds, the DRG was placed at the divergent junction of the fibrous bifurcation. DRGs were cultures in NeuralBasal A- medium with B27 supplement, $0.5 \mathrm{mM}$ L-glutamine and pen/ strep added (Gibco/Invitrogen) and $100 \mathrm{ng} \mathrm{ml}^{-1} \mathrm{NGF}$ (Sigma Aldrich). Cultures were maintained for $5 \mathrm{~d}$, with medium refreshed after Day 1 and Day 3. Cells were fixed with ice cold $2 \%$ paraformaldehyde (PFA) for $15 \mathrm{~min}$ at $4{ }^{\circ} \mathrm{C}$, then permeabilized for $10 \mathrm{~min}$ with
$0.1 \%$ TritonX-100. Cultures were blocked for $1 \mathrm{~h}$ in $5 \%$ Normal Goat Serum and 1\% BSA in a TRIS buffered solution (TBS), followed by a $16 \mathrm{~h}$ incubation with a mouse anti-b3-tubulin primary antibody (1:1000,Abcam), a triple wash in 1\% BSA TBS solution and a $16 \mathrm{~h}$ incubation with an Alexa 594 anti-mouse secondary antibody (1:1000, Invitrogen) with $1 \%$ Normal Goat serum. Scaffolds were then washed and mounted with Mowiol 4-88 with 2.5\% DABCO.

\section{Neurite measurement}

Fluorescence imaging of mounted DRG samples was performed with a BD Pathway 435 using a $10 \times$ objective. Automated Sholl Analysis was performed using ImageJ and the Sholl Analysis Plugin (ver.4.0.0) with a $20 \mu \mathrm{m}$ step size between concentric shell measurements. The resulting output was used to determine the 10 longest neurites, from which the average maximum length per sample was calculated.

Additional methodology information is available in supplementary material section. 


\section{Results and discussion}

\section{Electrode configuration focuses multiple polymer jets for controlled overlapping fiber deposition}

Traditional ESP process can be described as an electrostatic extrusion of a charged polymer solution from a supply needle towards a grounded collector. During this process, a polymer jet is formed which is initially straight but then enters an unstable whipping phase. This chaotic motion further extrudes the polymer jet and accelerates solvent evaporation to form solid polymer fibers [20]. To achieve more control over fiber deposition, this study manipulated the electrostatic field by using a grounded target comprised of millimeter wide electrodes with a centimeter-wide gap (figure 1(A), Inset: 'Collector'). The electric field across the gap is known to exert forces on the fibers during the whipping phase (figure 1(D), Inset: 'Gap'), leading to fiber alignment across the gap [21]. However, the use of millimeter wide electrodes in this configuration also achieved a focusing effect of the electric field in the orthogonal plane (figure $1(\mathrm{C})$ ) to further direct the evolution of the whipping phase.

ESP of one polymer solution with this arrangement led to the spatially confined deposition of aligned fibers across this gap (supplementary figure S2). Yet, this novel electrode architecture produced an electric field able to confine the simultaneous deposition of two ESP jets (figure 1(A), Inset 'Tandem Jets') to produce aligned parallel deposition of different fiber populations (figure 1(E)). To the best of our knowledge, this is the first report of concurrent and controlled deposition of different populations of aligned fibers with a welldefined region of overlap (figure $1(\mathrm{~F})$ ). When using optimized polymer solutions, as discussed below, this deposition was robust and repeatable with defined multi-fiber constructs consistently produced.

Despite initial mutual repulsion of the two jets during the stable jet phase (figure 1(A) Inset: 'Tandem Jets'), the focused electric field created by the narrow electrode geometry (figure 1(C)) provided sufficient force upon the fibers during the whipping phase to cause colocalized deposition. Fluorescence microscopy (figure $1(\mathrm{~F})$ ) revealed that the two regions were overlapping with no distinct boundary region between fiber populations. The fibers showed a relatively homogenous diameter and were well aligned, with no statistically significant differences between populations.

\section{Polymer properties dictate alignment for gap electrode ESP}

The degree of fiber alignment achieved was found to be dependent on the properties of the polymer solution. This provided the possibility of further control over fiber deposition and degree of multimodal complexity achievable.
The polymer solution has a dramatic impact on the ESP process in general, with many variables having a degree of interdependence. For example, the chosen solvent affects the solution viscosity but also the rate of evaporation during fiber formation, both of which alter ESP kinetics. Meanwhile, the polymer composition and molecular weight dictate the degree of solubility in a chosen solvent, the viscosity of the polymer solution, and can influence the rate of solvent evaporation [22].

To evaluate the impact of the solution properties on the T-ESP process, three copolymers from the PEOT/PBT family were selected. These polymers were synthesized using poly(ethylene oxide) (PEO) and poly(butylene terephthalate) (PBT) segments of specific lengths added in known proportions to produce a randomly distributed block copolymer. The PEO segment becomes terephthalated during the copolymer reaction and imparts a hydrophilic, amorphous phase, while the PBT introduces a hydrophobic, crystalline phase, such that the polymer properties can be tuned by controlling the length of the initial PEO segments and varying proportions of PEOT to PBT [23-25]. For example, a polymer with initial PEO segments of $300 \mathrm{Da}$ and a PEOT/PBT of 55/45 has the designation of 300PEOT55PBT45. Most importantly, this class of polymer is known to be soluble in the same solvent solution $\left(\mathrm{CHCl}_{3}\right.$ and HFIP) so that the final solutions are similar with respect to evaporation rate, polymer/solvent interactions, and solvent conductivity. Furthermore, different polymer compositions are available at similar molecular weights of approximately $50 \mathrm{kDa}$, providing a relatively consistent basis for comparison.

A distinguishing characteristic of the different selected polymers was the entanglement molecular weight $\left(M_{e}\right)$; this is a measure of the chain length between physical entanglement points of polymer chains in a solid bulk polymer. The three polymers selected were: a high entanglement (HighE) polymer with a composition of 300PEOT55PBT45 and a small $M_{e}$ of $250 \mathrm{Da}$; a midrange molecular entanglement (MidE) polymer (300PEOT70PBT30; $M_{e}: 450 \mathrm{Da}$ ); and 1000PEOT70PBT30, with an $M_{e}$ of $710 \mathrm{Da}$ and therefore called here LowE (low degree of molecular entanglement) [26]. These were prepared as $20 \% \mathrm{w} / \mathrm{v}$ polymer solutions in a solvent blend of $70 \%$ chloroform and 30\% hexafluoroisopropanol (HFIP; see supplementary material, tables S1 and S2 for details on polymers and solutions used).

Despite similar solvent and polymer concentrations, the HighE, MidE and LowE polymers solutions demonstrated different viscosities of 120, 125 and 388 centipoise $(\mathrm{cP})$, which produced fiber diameters of $0.86 \pm 0.28, \quad 0.74 \pm 0.34$, and $1.03 \pm 0.35 \mu \mathrm{m}$ respectively. Polymer solution viscosity is an indication of the degree of molecular entanglements in solution (not in bulk) [27]. $M_{e}$, along with viscosity and solution conductivity, has been cited as an important 
parameter in describing the polymer solutions used for ESP [28-30]. To eliminate this difference in polymer solution molecular entanglement as a potential variable, we included a LowE polymer solution with the same molecular weight but a lower polymer concentration to achieve a similar viscosity of $122 \mathrm{cP}$ (referred to as $\operatorname{LowE}_{m v}$, 'matched viscosity'; see table S2).

Unexpectedly, image analysis of fiber orientation found statistically significant decrease $(p<0.01)$ in alignment with increasing values of $M_{e}$. Using a score from 0 to 1 (random to aligned), alignments of $0.773 \pm 0.089,0.641 \pm 0.102$, and $0.33 \pm 0.077$ were measured for HighE, MidE, and LowE fibers, respectively. Consistent with this tread, the $\operatorname{LowE}_{m v}$ also resulted in low fiber alignment $(0.222 \pm 0.0698)$, indicating that viscosity, thus entanglement in the polymer solution, was not an effective predictor of fiber alignment. Instead, $M_{e}$ as a bulk polymer property appeared to be the predominant factor. Until now, $M_{e}$ has been used to describe the viscoelasticity of the polymer solution as it relates to the formation of 'beads-on-a string' fiber morphology formed in the initial stages of the polymer jet [28]. This finding suggested a potential role of $M_{e}$ on fiber evolution after they have returned to a bulk polymer state later in the whipping phase, with increasing bulk entanglement (HighE) improving electric field-induced fiber alignment.

\section{$M_{e}$ affects whipping phase evolution and fiber alignment}

Correlated with differences in fiber alignment, there were also observed changes in the whipping phase of the different polymers. The size and shape of the fiber deposition pattern onto the collector is direct evidence of how the whipping phase develops during the electrospinning process. The highly entangled HighE polymer deposited fibers along the length of the electrodes, indicating a smooth, elongated progression of the whipping phase. In comparison, the LowE polymer had fewer fibers along the length of the electrodes and a wider, more circular deposition in the electrode gap region, suggesting an abrupted, more focused evolution of the whipping fiber (supplementary material figure $\mathrm{S} 1$ ).

To account for this observed correlation between fiber alignment, macroscopic deposition pattern, and bulk molecular entanglement, it is proposed here that the fiber mechanical properties (as dictated by $M_{e}$ ) influence both fiber formation and the overall evolution of the whipping phase. While the whipping instability begins with an initial perturbation (bending) of the polymer jet, it has been reported that the fiber can also experience secondary and tertiary bending during the whipping phase. The consequence is that fibers are not necessarily smooth and straight, but can have a wavy, corrugated appearance [20]. It is proposed here that a fiber with a lower degree of bulk entanglement (a higher $M_{e}$ ) experiences more plastic deformation and accumulates non-axisymmetric residual stress during this final whipping phase [31-33], leading to a curled, corrugated fiber. In comparison, a fiber of a highly entangled polymer would have increased elasticity, resisting deformation, and would incur less residual stress to produce a straight fiber morphology. It should also be remarked that the long working distance used $(20 \mathrm{~cm})$ excludes the curled fibers to be the result of buckling [34].

We suggest that the electric field imposes orientation along the length of the fibers, such that a general 'global' alignment is achieved; this is evidenced by the fact that the observed fiber orientation is not entirely random, especially compared to fibers deposited on a flat collector plate (see table S5). However, the resulting microscopic alignment of the fiber also depends on how corrugated the fibers have become, with higher $M_{e}$ polymers producing fibers with some degree of alignment but with increasing disorder. Since strain and entanglement can also be affected by differences in molecular weight, the use of these particular polymers proved essential in identifying the role of $M_{e}$ [35].

These additional plastic perturbations during fiber formation are also suggested to disrupt the whipping profile development, accounting for the observed differences in the elongated deposition of fibers. An elongated whipping profile may also increase the degree of microscopic fiber alignment of the HighE polymer fibers by improving global fiber alignment and effectively stretching out any fiber perturbations.

\section{Heterogeneous fiber patterns can be tuned by adjusting polymer solution properties}

T-ESP was extended to produce heterogeneous fiber populations, using the HighE and the LowE polymers as extremes in terms of both fiber size and fiber alignment. Again using a score from 0 to 1 (random to aligned), heterogeneous T-ESP produced the expected regions of aligned HighE fibers $(0.699 \pm 0.104)$, less aligned LowE fibers $(0.401 \pm 0.06)$, and an overlapping region between the two populations. Unexpectedly, this also resulted in a fiber deposition pattern with an extreme spatial bias, where the LowE fibers now shifted to the edge of the deposition region (figure 2(A)).

Aiming to understand this bias, a comparison was made between the profiles of the tandem jets when two identical HighE solutions were used or when HighE and LowE polymer solutions were tandem-spun together. This revealed that T-ESP with identical polymer solutions produced stable jets of equal length (figure 1(A) Inset: 'Tandem Jets') while heterogeneous T-ESP exhibited different lengths, with the HighE solution initiating the chaotic whipping phase at an earlier stage (i.e. a shorter stable jet) compared to the LowE solution (figure 2(B)). Because the whipping 


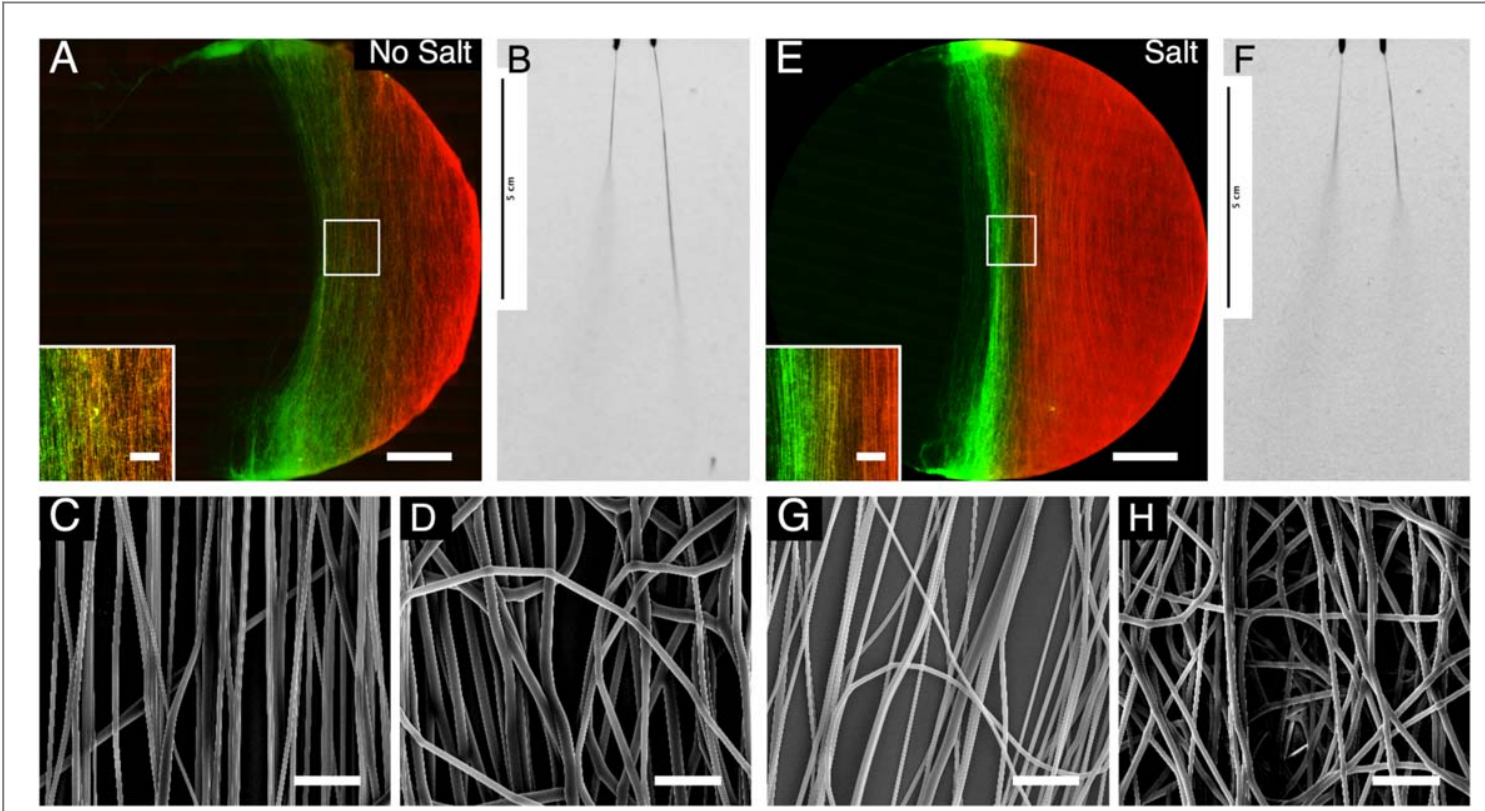

Figure 2. Heterogeneous Tandem ESP Scaffolds. (A) Tandem ESP fibers of HighE (green) and LowE (red) produced a biased pattern of fiber deposition, though an overlapping gradient region between the two fiber populations was still maintained (inset). (B) The whipping jet profiles reveal that the LowE solution initiates whipping later compare to the HighE solution (scale bar: $5 \mathrm{~cm})$. (C) The HighE fibers had a diameter of $0.723 \pm 0.261 \mu \mathrm{m}$ and were well aligned $(0.699 \pm 0.061)$. (D) LowE fibers were significantly larger in diameter $(1.61 \pm 0.345 \mu \mathrm{m}, p<0.01)$, and were less aligned $(0.397 \pm 0.076, p<0.01)$. (E) Tandem electrospun fibers of HighE polymer solution and $\mathrm{LowE}^{+}$solution produced a centered pattern of fiber deposition with an overlapping gradient region between the two fibers also evident (inset). (F) The whipping profiles show that the HighE jet (left) and the LowE ${ }^{+}$jet have similar stable jet lengths (scale bar: $5 \mathrm{~cm}$ ). (G) HighE had fiber diameters of $0.859 \pm 0.311 \mu \mathrm{m}$ and were relatively aligned $(0.551 \pm 0.106)$. (H) LowE fibers were smaller in size, though still significantly larger than HighE fibers $(0.865 \pm 0.164 \mu \mathrm{m}, p>0.01)$, and maintained the same approximate alignment as the original LowE fibers $(0.397 \pm 0.076, p<0.01)$. (Scale bars: A, E $2 \mathrm{~mm} ;$ Inset $500 \mu \mathrm{m} ; \mathrm{C}, \mathrm{D}, \mathrm{G}, \mathrm{H}$, $10 \mu \mathrm{m})$.

phase of the HighE evolved along the length of the electrodes (as described above), and therefore was exposed to more of the electrode area, the initial hypothesis was that the HighE fibers were subject to more overall electrostatic force during their flight time compared to the more localized LowE fibers, such that the HighE jet is preferentially attracted while LowE fibers are deflected to create the observed spatial bias.

To test this hypothesis, the effective force experienced by the LowE fibers was increased by adding $0.05 \mathrm{mg} \mathrm{ml}^{-1}$ of salt $(\mathrm{NaCl})$ to the LowE polymer solution (referred to hereafter as LowE ${ }^{+}$) [30]. The LowE ${ }^{+}$ solution was confirmed to have increased conductivity (supplementary table S2) and the increased surface charge of the LowE $\mathrm{E}^{+}$fibers was evident from a much shorter stable jet region (supplementary figure 2(F)); this follows previous reports, where the addition of surface charge hastens the onset of the chaotic whipping phase [36]. With an increase in electrostatic force and with each polymer now having roughly equal stable jet length, the end result was a more centered distribution of HighE and LowE ${ }^{+}$fibers (figure 2(E)). The LowE ${ }^{+}$fibers were also smaller, consistent with a previous report on the effect of salt [37], a no change in the degree of alignment between the salt and salt-free condition was observed.

Additional experiments confirmed the role of electrostatic force versus the possible influence of stable jet length. By substituting the LowE solution with the lower viscosity LowE $\mathrm{E}_{m v}$ solution, the initiation of the whipping phase was now earlier compared to the HighE solution (supplementary figures S5(E), (F)); according to Hohman et al [38], the onset of whipping occurs once the polymer jet reaches a critical radius, with less viscous solutions reaching this stage earlier in the fluidic jet evolution. This still resulted in a biased fiber pattern, with HighE remaining dominant on the target substrate (supplementary figure S5(A)). Examining the later stages of jet evolution revealed that HighE fibers experienced a final pull towards the target area (supplementary figure S5(K)), further supporting the hypothesis that the elongated whipping profile of the HighE fibers resulted in greater electrostatic attraction. Adding salt to the LowE $\mathrm{E}_{m v}$ solution (hereafter Low $\mathrm{E}_{m v}^{+}$), evidence of increased surface charge was again observed by a much shorter stable jet length (approximately $1.5 \mathrm{~cm}$ ) and a more centered distribution of tandem fiber deposition with a defined region of overlap (supplementary figure S7). The resulting LowE $\mathrm{E}_{m v}^{+}$fibers were now equal in diameter $(0.774 \pm 0.287 \mu \mathrm{m})$ to the tandem-spun HighE fibers $(0.859 \pm 0.311 \mu \mathrm{m}, p<0.01)$ but maintained a reduced degree of alignment $(0.247 \pm 0.0586$, scaled from 0 -random to 1-aligned). In summary, tuning polymer solutions used for heterogeneous T-ESP makes possible fiber constructs with both distinct fiber alignments and tailored distribution patterns. 

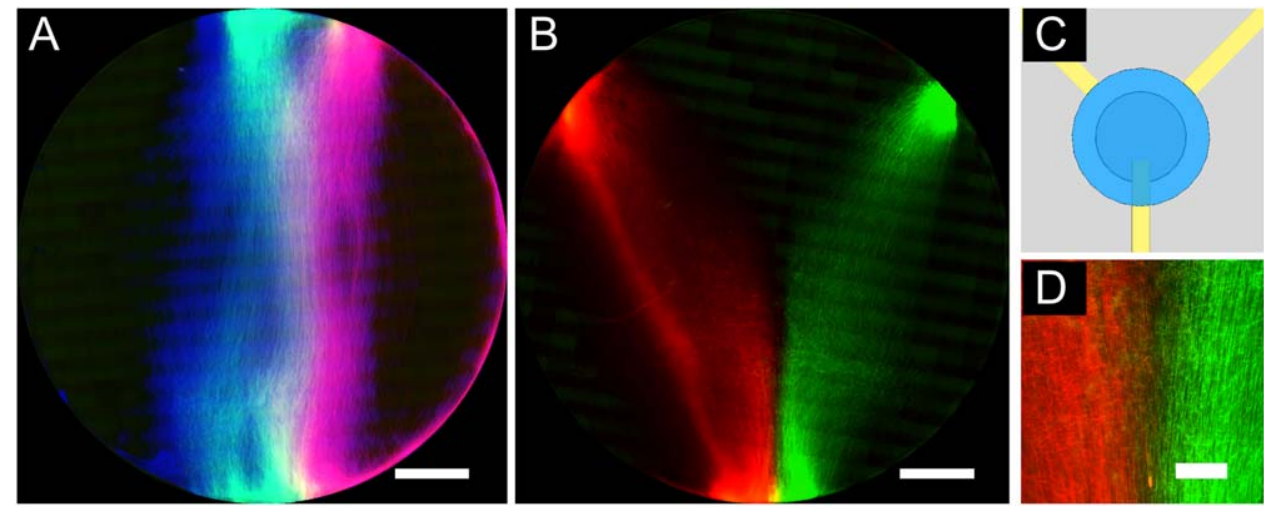

Figure 3. Triple ESP and Divergent Tandem ESP. (A) An example of focused aligned electrospinning with 3 separate HighE solutions simultaneously (scale bar: $2 \mathrm{~mm}$ ). A different dye was added to each solution (see Methods section). The blue autofluorescence of HighE was enhanced by adding Pyrene, though the residual blue autofluorescence of the other fiber populations modified the typical green and red dye colors to mint and pink, respectively. (B) The result of tandem ESP on an 'epsilon' gap electrode arrangement (shown in C), producing a region of overlapping fibers (scale bars, B: $2 \mathrm{~mm}$; D: $500 \mu \mathrm{m}$ ) which then diverge. Further details of the needle and target collector arrangements for these patterns are described in supplementary material figure S9. Fiber diameter is $0.788 \pm 0.279 \mu \mathrm{m}$, consistent with previous HighE ESP fibers.

\section{Robust tandem deposition and complex T-ESP fiber patterning}

Once the optimization of T-ESP was established, the resulting tandem deposition was observed to be highly robust. Scaffolds were produced one at a time, taking approximately $5 \mathrm{~min}$ per scaffolds and an estimated $100 \%$ success rate. Given the robust nature of this approach, throughput could potentially be increased via deposition onto a narrow rotating disc collector [39], though care should be taken to avoid extraneous grounding points which may disrupt the electric focusing effect observed here.

Given the relative stability of this process, we extended this strategy to include multi-jet spinning to create different patterns of fiber deposition. For example, moving from two to three jets resulted in the simultaneous overlapping deposition of three populations of aligned fibers (figure $3(\mathrm{~A})$ ). When optimizing this process, achieving uninterrupted jet stability for all three polymers required an inter-needle separation of $5 \mathrm{~cm}$, for a distance of $10 \mathrm{~cm}$ between the two outer needles. The outer polymer jets still converged onto the narrow gap electrode. However, this inter-needle spacing was an intrinsic limit to our system; farther separation resulted in fiber deposition on extraneous grounding points located within the electrospinning chamber. With a complete redesign of our current electrospinning apparatus, one could expect successful T-ESP processing with farther needle separations.

The target electrode arrangement was further altered to achieve a divergent pattern of deposition (figure 3(C); supplementary figure S9 for electrode details). Figure 3(B) shows two fiber populations which have both deposited onto a 'shared' bottom electrode in an aligned, overlapping manner (figure 3(D)). These fibers then diverged and separated, with the left polymer fibers (red) oriented towards the left upper electrode and the right polymer fibers (green) directed towards the right electrode. This creates a unique fiber pattern with a degree of complexity not yet seen for ESP. All patterns described could also be replicated by performed T-ESP with a $5 \%$ solution of $\mathrm{PEO}$ in $\mathrm{dH}_{2} \mathrm{O}$, confirming that this process is compatible with other polymers and solvent (figure supplementary S8).

\section{T-ESP applications}

This new fabrication approach has an immediate application in the field of tissue engineering and regenerative medicine. The design of fibrous scaffolds follows a common biomimetic principle, with the intent of achieving an appropriate cell response by imitating the natural fibrous extracellular matrix found in the body. Current ESP scaffold can be tailored to elicit specific cell behavior, but fibers are typically modified homogeneously. However, this does not reflect the heterogeneous cell populations found in vivo. The possibility to create tissue scaffolds with purposely-designed heterogeneity may enhance scaffold effectiveness, exacting spatial control of cell response imparted by the properties of respective fiber types. Furthermore, bifurcated branching structures are ubiquitous throughout the body, including, but not limited to, nerves, vasculature, pulmonary and breast tissue [40-43]. The ability to make fibrous scaffolds as described in this work provides a promising tool for studying these types of biological systems, such as evaluating cellular decision-making processes at an ECM-like bifurcation, as well as for the development of tissue engineering solutions.

To show the utility of T-ESP fiber tissue scaffolds for the study of presenting a biological system with a defined decision point, we examined the impact of a heterogeneous divergent scaffold of HighE fibers and 

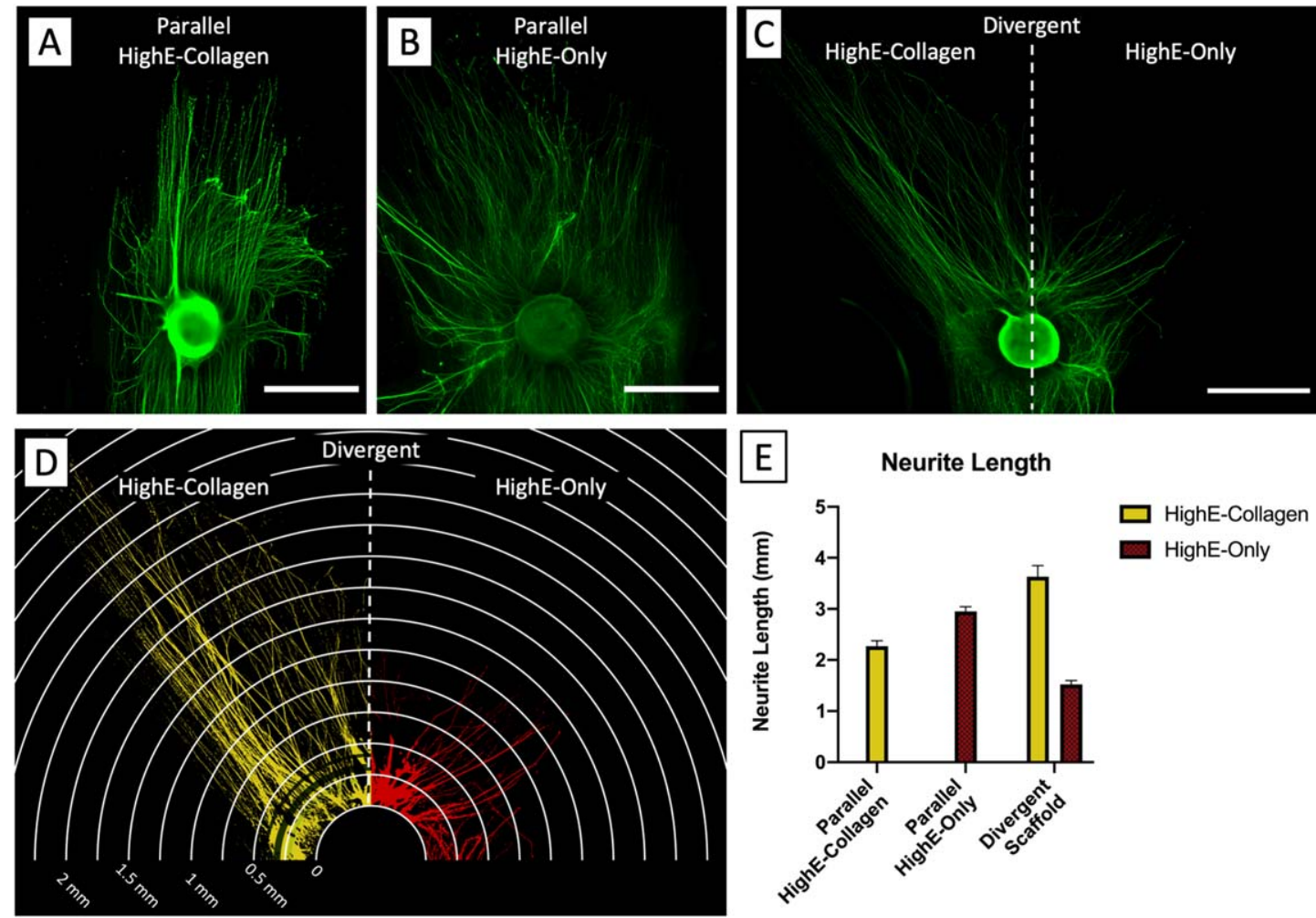

Figure 4. Spatially modulated neurite outgrowth on a divergent heterogeneous Tandem ESP scaffold. An explanted DRG (large, round structure) grown on aligned fiber scaffolds of either HighE-Collagen (A) or HighE-Only (B) do not exhibit such a dramatic different in neurite length, with measurements of $2232 \pm 74 \mu \mathrm{m}$ and $2953 \pm 88.7 \mu \mathrm{m}$ respectively. In contrast, a divergent T-ESP pattern (C) exhibits a distinctive different between the two fiber types, comprised of HighE-collagen fibers diverging to the left half labeled, 'HighE-Collagen', and HighE fibers diverging to the right half labeled 'HighE-Only'. With a DRG explant placed at the bifurcation of the two fiber types, the resulting growth pattern shows a clear preference for the 'HighE-Collagen' domain with an average neurite length of $3632 \pm 217.4 \mu \mathrm{m}$ versus the 'HighE-only' with a length of $1522 \pm 77.3 \mu \mathrm{m}$ (scale bar $1 \mathrm{~mm}$ ). The divergent pattern lends itself to automated Sholl Analysis (D), whereby the evaluation of the 10 longest neurites (E) can quickly be determined to examine the impact of creating a clear decision point within a fibrous scaffold.

an HighE-collagen blended fibers on neurite outgrowth (figure 4). An earlier study showed modulated neurite growth on ESP scaffolds of $\mathrm{PCL} /$ collagen blended fibers compared to scaffolds of PCL-only fibers [44]. For our particular polymer system, an explanted dorsal root ganglion (DRG) grown on aligned fiber substrates that were homogeneously HighE-collagen or HighE-only fibers exhibited comparable levels of neurite growth, with a neurite length of $2232 \pm 74 \mu \mathrm{m}$ and $2953 \pm 88.7 \mu \mathrm{m}$, respectively (figures 4(A), (B)). This suggests that for the HighE polymer, the addition of collagen has limited impact on neurite growth.

In contrast, our divergent scaffold resulted in notably longer neurite extension on the HighEcollagen fibers compared to HighE-only fibres (figure 4(C)). Neurites also followed the fiber orientation, creating a divergent growth pattern that facilitated rapid assessment of the competitive outgrowth via automated Sholl Analysis (figure 4(D), supplementary figure S13). The plotted length of maximum neurite growth for each condition (figure 4(E)) clearly shows that the HighE-collagen portion of the divergent scaffold achieved a neurite length of $3632 \pm 217.4 \mu \mathrm{m}$ while the HighE-only fibers supported approximately half the amount of growth, with a length of $1522 \pm 77.3 \mu \mathrm{m}$. In contrast, a divergent scaffold consisting of HighE-only fibers exhibits no visible bias (supplementary figure S14), indicating that it is the combination of the divergent fiber pattern and the organized fiber heterogeneity produced by the T-ESP process that stimulates this defined growth pattern. Overall, this suggests that neurites will readily grow on oriented nanofibrous scaffolds even in the absence of a preferred option. But if given a choice, a clear preference can be determined. This presents unique biofabrication possibilities for neural tissue engineering, such as generation of a nanofibrous scaffold for the spatially selective promotion of different neural subpopulations or a general tissue engineering approach for sorting of a heterogeneous cell population.

A gradient of fiber alignment could also spatially modulate cell morphology and, thus, creating a gradient of cell function. The spatial control provided by the T-ESP process also provides a means to investigate 
the fabrication tolerances required in order to elicit such a graded response. Figure S10 shows the modulation of Schwann cell morphology on a T-ESP scaffold of aligned HighE fibers and less aligned LowE $\mathrm{E}_{m v}^{+}$fibers, where such differences in Schwann cell morphology are known to modulate their production of neurotrophic factors [45].

The application of this technology is not limited to the field of Tissue Engineering. Initial trials have also employed tandem scaffolds to separate mixed solutions on the basis of fiber affinity (supplementary figure S11), showing promise in the field of Separation Technologies. This fabrication technique can be used to prepare heterogeneous catalytic or filtration substrates or high surface area nanofibrous cathode/ anode electrode configurations able to sequester specific analytes.

\section{Conclusions}

Until now, the ease and promising characteristics of ESP has found limited applicability due to limitations in the ability to control and customize the resulting fiber meshes. This work presents the highly accessible T-ESP approach, providing the versatility to create an array of micro- and nano-scale fibrous constructs with complex, ordered patterns. At the same time, this work provides general considerations for optimal polymer selection as well as furthering the understanding of the ESP process. Though a promising tool for tissue engineering applications, this approach holds potential for other fields. The methodology presented here further extends the growing arsenal of ESP solutions to create increasingly complex polymer fiber constructs in a simple manner to address the growing demands of new material and engineering challenges.

\section{Acknowledgments}

The authors would like to thank Dr Maqsood Ahmed, Dr Alvaro Gomez Marin, and Dr Hugo Fernandez for enlightening discussion during the experimental work and Mijke Buitinga for assistance in manuscript preparations. This work was partly funded by the Natural Science and Engineering Research Council (NSERC) of Canada and by funding received from the European Community's Seventh Framework Programme (FP7/2007-2013) under MERIDIAN (grant agreement $\mathrm{N}^{\circ} 280778$ ).

\section{Conflict of Interest}

Authors declare no conflicts of interest.

\section{ORCID iDs}

Paul Wieringa (iD https://orcid.org/0000-00023290-5125

Lorenzo Moroni (iD https://orcid.org/0000-0003$1298-6025$

\section{References}

[1] Teo W and Ramakrishna S 2006 A review on electrospinning design and nanofibre assemblies Nanotechnol. Inst. Phys. 17 89-106

[2] Ramakrishna S, Fujihara K, Teo W, Yong T and Ramaseshan R 2006 Electrospun nanofibers: solving global issues Mater. Today 9 40-50

[3] Liu W, Thomopoulos S and Xia Y 2012 Electrospun nanofibers for regenerative medicine Adv. Healthc. Mater. 1 10-25

[4] Gopal R, Kaur S, Ma Z, Chan C, Ramakrishna S and Matsuura T 2006 Electrospun nanofibrous filtration membrane J. Membr. Sci. 281 581-6

[5] Thavasi V, Singh G and Ramakrishna S 2008 Electrospun nanofibers in energy and environmental applications Energy Environ. Sci. 1205

[6] Ding B, Wang M, Wang X, Yu J and Sun G 2010 Electrospun nanomaterials for ultrasensitive sensors Mater. Today 13 16-27

[7] Li D and Xia Y 2004 Electrospinning of nanofibers: reinventing the wheel? Adv. Mater. 16 1151-70

[8] Grasl C, Arras M M L, Stoiber M, Bergmeister H and Schima H 2013 Electrodynamic control of the nanofiber alignment during electrospinning Appl. Phys. Lett. 10205311

[9] Varesano A, Carletto R A and Mazzuchetti G 2009 Experimental investigations on the multi-jet electrospinning process J. Mater. Process. Technol. 209 5178-85

[10] Wang Y, Li H, Wang G, Yin T, Wang B and Yu Q 2010 Electrospinning of polymer nanofibers with ordered patterns and architectures J. Nanosci. Nanotechnol. 10 1699-706

[11] Li D, Wang Y and Xia Y 2004 Electrospinning nanofibers as uniaxially aligned arrays and layer-by-layer stacked films $A d v$. Mater. 16361-6

[12] Kim G, Cho Y and Kim W 2006 Stability analysis for multi-jets electrospinning process modified with a cylindrical electrode Eur. Polym. J. 42 2031-8

[13] Gupta P and Wilkes G L 2003 Some investigations on the fiber formation by utilizing a side-by-side bicomponent electrospinning approach Polymer 44 6353-9

[14] Kidoaki S, Kuen K and Matsuda T 2005 Mesoscopic spatial designs of nano- and micro ber meshes for tissue-engineering matrix and scaffold based on newly devised multilayering and mixing electrospinning techniques Biomaterials 26 37-46

[15] Skotak M, Ragusa J, Gonzalez D and Subramanian A 2011 Improved cellular infiltration into nanofibrous electrospun cross-linked gelatin scaffolds templated with micrometersized polyethylene glycol fibers Biomed. Mater. 6 1-20

[16] Chang C, Limkrailassiri K and Lin L 2008 Continuous nearfield electrospinning for large area deposition of orderly nanofiber patterns Appl. Phys. Lett. 93123111

[17] Hutmacher D W and Dalton P D 2011 Melt electrospinning Chem. Asian J. 644-56

[18] Rezakhaniha Ret al 2012 Experimental investigation of collagen waviness and orientation in the arterial adventitia using confocal laser scanning microscopy Biomech. Model. Mechanobiol. 11 461-73

[19] Ayres C et al 2006 Modulation of anisotropy in electrospun tissue-engineering scaffolds: analysis of fiber alignment by the fast Fourier transform Biomaterials 27 5524-34

[20] Reneker D H, Yarin A L, Fong H and Koombhongse S 2000 Bending instability of electrically charged liquid jets of polymer solutions in electrospinning J. Appl. Phys. 874531

[21] Li D, Wang Y and Xia Y 2003 Electrospinning of polymeric and ceramic nanofibers as uniaxially aligned arrays Nano Lett. 3 $1167-71$ 
[22] Song JY, Wang Y Y and Wan C C 1999 Review of gel-type polymer electrolytes for lithium-ion batteries J. Power Sources 77 183-97

[23] Bulstra S K, Geesink R G, Bakker D, Bulstra T H Bouwmeester S J and van der Linden A J 1996 Femoral canal occlusion in total hip replacement using a resorbable and flexible cement restrictor J. Bone Joint Surg. Br. 78 892-8

[24] Bakker D, van Blitterswijk C A, Hesseling S C, Koerten H K, Kuijpers W and Grote J J 1990 Biocompatibility of a polyether urethane, polypropylene oxide, and a polyether polyester copolymer. A qualitative and quantitative study of three alloplastic tympanic membrane materials in the rat middle ear J. Biomed. Mater. Res. 24 489-515

[25] Nandakumar A, Fernandes H, de Boer J, Moroni L, Habibovic P and van Blitterswijk C A 2010 Fabrication of bioactive composite scaffolds by electrospinning for bone regeneration Macromol. Biosci. 10 1365-73

[26] Moroni L, de Wijn J R and van Blitterswijk C A 2005 Threedimensional fiber-deposited PEOT/PBT copolymer scaffolds for tissue engineering: influence of porosity, molecular network mesh size, and swelling in aqueous media on dynamic mechanical properties J. Biomed. Mater. Res. 75 957-65

[27] Kavassalis T A and Noolandi J 1989 Entanglement scaling in polymer melts and solutions Marcomolecules. 22 2709-20

[28] Shenoy S L, Bates W D, Frisch H L and Wnek G E 2005 Role of chain entanglements on fiber formation during electrospinning of polymer solutions: good solvent, non-specific polymerpolymer interaction limit Polymer 46 3372-84

[29] Tang C, Ozcam A E, Stout B and Khan S A 2012 Effect of pH on protein distribution in electrospun PVA/BSA composite nanofibers Biomacromolecules. 13 1269-78

[30] Angammana C J and Jayaram S H 2011 Analysis of the effects of solution conductivity on electrospinning process and fiber morphology IEEE Trans. Ind. Appl. 47 1109-17

[31] Yarin A L, Koombhongse S and Reneker D H 2001 Bending instability in electrospinning of nanofibers J. Appl. Phys. 893018

[32] Bartczak Z2010 Effect of chain entanglements on plastic deformation behavior of ultra-high molecular weight polyethylene J. Polym. Sci. B 48 276-85
[33] Wissuchek D J, Ponader C W and Price J J 1999 Analysis of residual stress in optical fiber Proc. SPIE 3848 34-43

[34] Han T, Reneker D H and Yarin A L 2007 Buckling of jets in electrospinning Polymer 48 6064-76

[35] McLeish T C B, Plummer C J G and Donald A M 1989 Crazing by disentanglement : non-diffusive reptation Polymer 30 1651-5

[36] Hohman M M, Shin M, Rutledge G and Brenner M P 2001 Electrospinning and electrically forced jets: I. Stability theory Phys. Fluids 132201

[37] Jean-Gilles R et al 2010 Novel modeling approach to generate a polymeric nanofiber scaffold for salivary gland cells J. Nanotechnol. Eng. Med. 131008

[38] Hohman M M, Shin M, Rutledge G and Brenner M P 2001 Electrospinning and electrically forced jets: II. Applications Phys. Fluids 132221

[39] Theron A, Zussman E and Yarin A L 2001 Electrostatic fieldassisted alignment of electrospun nanofibres Nanotechnology 12384-90

[40] Nguyen Q T, Sanes J R and Lichtman J W 2002 Pre-existing pathways promote precise projection patterns Nat. Neurosci. 5 861-7

[41] Nelson C M, Vanduijn M M, Inman J L, Fletcher D A and Bissell M J 2006 Tissue geometry determines sites of mammary branching morphogenesis in organotypic cultures Science 314 298-300

[42] De Smet F, Segura I, De Bock K, Hohensinner P J and Carmeliet P 2009 Mechanisms of vessel branching: filopodia on endothelial tip cells lead the way Arterioscler. Thromb. Vasc. Biol. 29 639-49

[43] Lu P and Werb Z 2008 Patterning mechanisms of branched organs Science 322 1506-9

[44] Schnell E et al 2007 Guidance of glial cell migration and axonal growth on electrospun nanofibers of poly-epsiloncaprolactone and a collagen/poly-epsilon-caprolactone blend Biomaterials 28 3012-25

[45] Masaeli E et al 2013 Fabrication, characterization and cellular compatibility of poly(hydroxy alkanoate) composite nanofibrous scaffolds for nerve tissue engineering PLoS One 8 e57157 